\author{
Garczynski, E. 28 \\ Holly, A. 28, 50 \\ Lachner, G. 18, 28 \\ Lamertz, C. 75 \\ Lieb, R. 8, 58, 67, 75 \\ Nelson, C.B. 5, 18, 42 \\ Perkonigg, A. 8, 18, 28, 58 \\ Pfister, H. 28, 67 \\ Schuster, P. 28, 75 \\ Türk, D. 28 \\ Wittchen, H.-U. 5, 8, 18, 28, 42, 50, 58, 67, 75 \\ Wunderlich, U. 28
}

\title{
Subject Index Vol. 4, No. 1-2, 1998
}

Abuse of prescription drugs 67

Adolescents 50,67

Age at onset 58

Alcohol 8

- abuse 50

- dependence 50

Amphetamines 75

Analgesics/opioids 67

Assessment 18

Comorbidity 42

Dependence on prescription drugs 67

Diagnostic instruments 18, 28

Diagnostic reliability 28

Ecstasy 75

Epidemiology 8, 18, 42, 67

Hallucinogens 75
Illicit drugs 8

- $\quad$, use, abuse and dependence 58

Nicotine 8

- dependence 42

Prevalence 58, 67, 75

Prospective designs 18

Psychotropic medicines 8

Reliability 28

Sedatives 67

Smoking 42

Stimulants 67,75

Substance abuse 75

- use, abuse 8

- - disorders 18, 28

Symptom progression 50

Use of prescription drugs 67 